\title{
Cause of Ascending Aortic Dilatation in Patients with Bicuspid Aortic Valves: The Final Link
}

\author{
Francis Robicsek $^{1}$ Jeko M. Madjarov ${ }^{1}$ Robert F. Padera Jr. ${ }^{2}$ \\ 1 Department of Thoracic and Cardiovascular Surgery, Sanger Heart \\ and Vascular Institute, Carolinas Medical Centre, Charlotte, \\ North Carolina, United States \\ 2 Department of Pathology, Brigham and Women's Hospital, \\ Boston, Massachusetts, United States \\ Address for correspondence Francis Robicsek, MD, PhD, The \\ Department of Thoracic and Cardiovascular Surgery, Sanger Heart \\ and Vascular Institute, Carolinas Medical Centre, Charlotte, NC, \\ United States \\ (e-mail: Theresa.R.Johnson@atriumhealth.org; \\ francis.robicsek@atriumhealth.org).
}

Thorac Cardiovasc Surg 2020;68:277-280.

\begin{abstract}
Keywords

- aortic dilatation

- bicuspid aortic valves

Background This article discusses about the cause of dilatation of the ascending aorta in patients with congenitally bicuspid aortic valves (CBAVs). Some members of the profession believe that it is genetic, while others attribute it to turbulence. The author previously presented in vitro data proving that CBAVs are inherently morphological stenotic, even in the absence of measurable gradient and clinical symptoms. This article reinforces the view that the dilatation of the ascending aorta in patients with CBAV represents a "poststenotic dilatation" by demonstrating that the structure of the aortic wall is normal in infants who have CBAV as a sole cardiac anomaly.

Methods The aortic wall was studied in newborns who had CBAV as the sole cardiovascular anomaly and in those where CABV was associated with other inborn cardiovascular defects.

Results We found that in patients where CBAV is the sole cardiovascular anomaly, aortic structure is normal at birth, but abnormal if additional cardiovascular anomalies are present.

Conclusions Dilatation of the ascending aorta in patients, where CBAV is the only cardiovascular anomaly, is caused by turbulence (poststenotic dilatation). If additional cardiovascular anomalies are present, this process may be exaggerated by genetic abnormalities of the aortic wall.
\end{abstract}

\section{Introduction}

Dilatation of the ascending aorta in patients with congenitally bicuspid aortic valves (CBAV) is a well-known phenomenon, which often requires a major surgical intervention. Most members of our profession believe that this dilatation is caused by inherent weakness of the aortic wall, ${ }^{1}$ which should be treated with radical surgery, even if the dilatation is mild, or even nonexistent. ${ }^{2,3}$

We believe that the condition (CBAV) is analogous with "poststenotic dilatation" (PD)-a curious enlargement of a vascular segment distal to a stenotic area. This phenomenon

received

August 14, 2018

accepted after revision

October 8, 2018

published online

November 29, 2018

was already recognized by the great Italian anatomist Morgagni ${ }^{4}$ and in modern times clinically studied by Halsted ${ }^{5}$ and Holman. ${ }^{6}$ They postulate that PD is acquired, caused by mechanical damage by the turbulent blood flow streaming through the stenotic area (-Figs. 1-2). This has been further studied by us in $1954{ }^{7,8}$ Later we extended the theory of PD also to dilatation of the ascending aorta which occurs in patients with CBAV. , $^{9,10}$

The principal arguments that CBAV is associated with congenital weakness of the ascending aortic wall, is that, disarray and fragmentation of the elastic fibers, as well as disappearance of muscular elements and disturbed collagen

(c) 2020 Georg Thieme Verlag KG Stuttgart . New York
DOI https://doi.org/ 10.1055/s-0038-1675762. ISSN 0171-6425. 

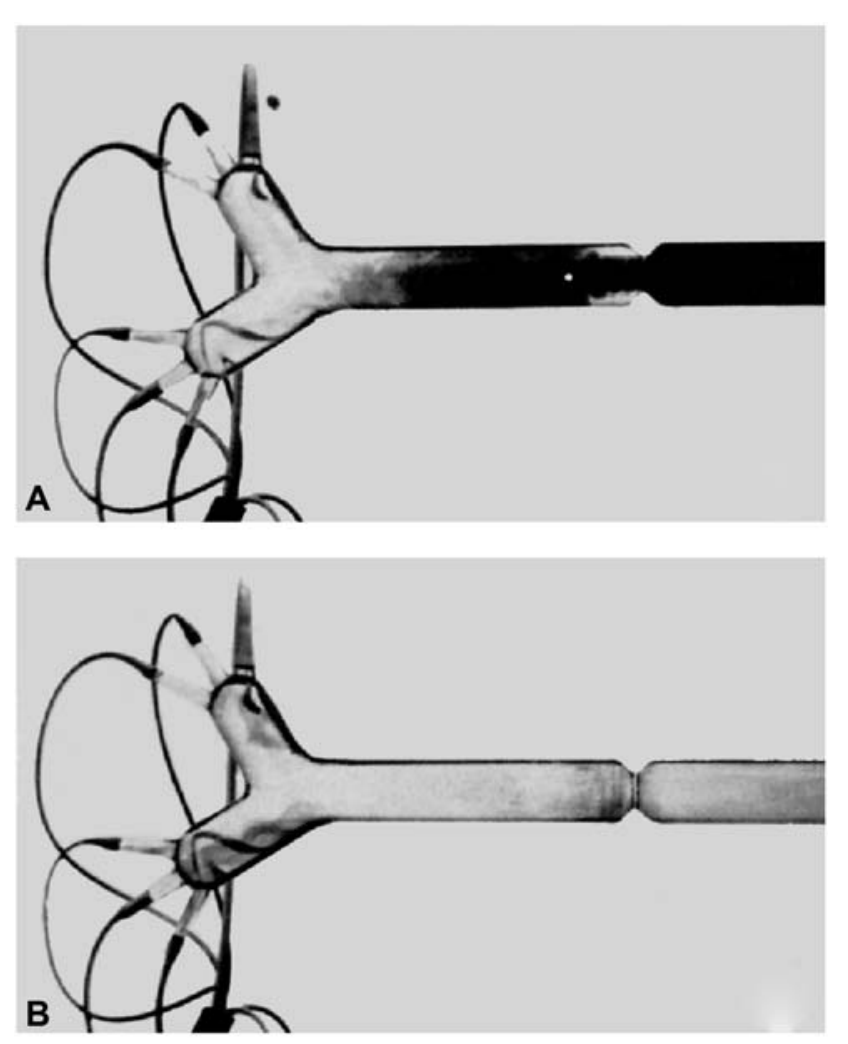

Fig. 1 In the in vitro model, the flow continues constricted past the stenosis (A) and vortices continues to linger in the poststenotic segment (B). (Adapted from Robicsek. ${ }^{8}$ )

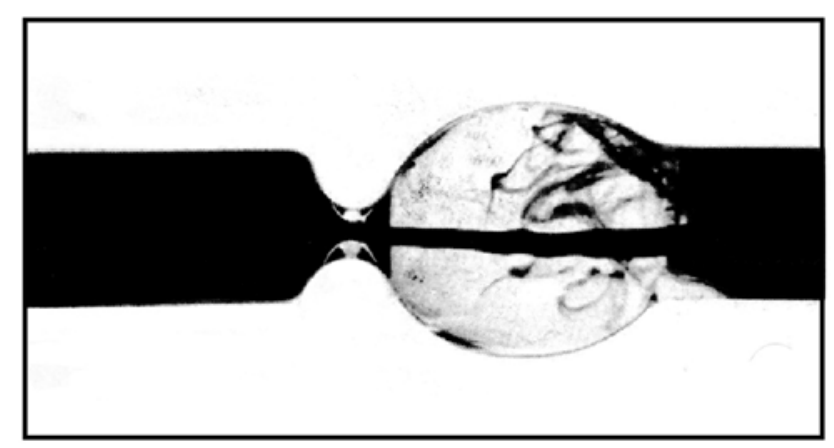

Fig. 2 Vortex formation is extensive if the poststenotic segment is dilated. (Adapted from Robicsek. ${ }^{7}$ )

structure, frequently occurs in individuals with CBAVs, regardless of presence or absence of ascending aortic dilatation. ${ }^{11}$ It should be noted that in most of these studies the aortic wall was sampled from grown individuals. Also, similar, if not identical, changes occur in patients with arteriosclerotic aortas or in those with Marfan syndrome, ${ }^{1,11,12}$ especially if the biopsy was taken from a dilated segment.

Our previous observations ${ }^{9,10}$ also challenge the concept that there are "non-stenotic" CBAVs. In a patient with such condition, the bicuspid valve may not generate any transvalvular pressure gradient; the patient may be asymptomatic, albeit every bicuspid aortic valve is physiologically stenotic. These studies were made in, the hearts of young individuals with CBAVs, ages 16 to 28 years, who had no cardiac symptoms and who died of non-cardiac causes. We found all the bicuspid aortic valves to be 30 to $50 \%$ stenotic (-Fig. 3). This is not surprising, if somebody considers the functional mechanism of the bicuspid aortic valve, where dynamically thinking, some degree of stenosis is unavoidable to assure adequate opening and closure. This "stenosis" may be only relative, considering that the annuli of bicuspid aortic valves were found to be larger than those of normal tricuspid aortic valves. ${ }^{13}$ Ergo propter it is logical to conclude that a bicuspid aortic valve, even if the patient is asymptomatic and lacks significant aortic gradient, may generate enough turbulence to damage the wall of the ascending aorta. The presence of significant turbulence in asymptomatic patients is also supported by the presence of a murmur and often a palpable thrill, over the aorta. ${ }^{9,10}$

Notably, abnormal flow patterns also occur in patients with stenosis of their initially normal trileaflet aortic valve. In these patients, the ascending aortic dilatation/aneurysm is usually symmetrical "pear" or "teardrop" shaped, while in patients with CBAV the dilatation is convex toward the right anterolateral aspect where degenerative changes of the media were also found to be the most severe. Studying the issue with computer simulation, we found that the direction of the flow through the CBAV, due to asymmetry of the valve leaflets, is not central but directed in the anterolateral direction. Stress overload was also measured to be highest at the same location in patients with "normally" functioning CBAVs. $^{14}$

The fact that not every patient with bicuspid aortic valve develops ascending aortic dilatation may be explained with individual variation in the strength of the elastic elements in the aortic wall.

We realize that while these arguments are valid indeed, they still do not provide an absolute proof of our postulate, that is, turbulence is the main reason for an ascending aortic dilatation in patients with bicuspid aortic valves. The issue, therefore, was further investigated.

\section{Methods}

We began our additional observations on the aorta of a 5 years old child (-Fig. 4) who died of pneumonia and who had bicuspid aortic valve. The histological examination of the ascending aorta showed not only the disruption of the elastic fibers but also turbulence-induced plaque formation, which was readily visible even by the naked eye. After that, we histologically examined the aortic roots obtained from infants with CBAV who expired within a week of their birth. We were able to collect four such specimens. Two infants with normal trileaflet aortic valves served as controls. Samples were stained for hematoxylin-eosin and for elastic fibers. According to the findings, the specimens were divided in three groups.

\section{Results}

Group 1 was composed of two specimens, where the full autopsy revealed CBAV as the sole cardiovascular anomaly. In 

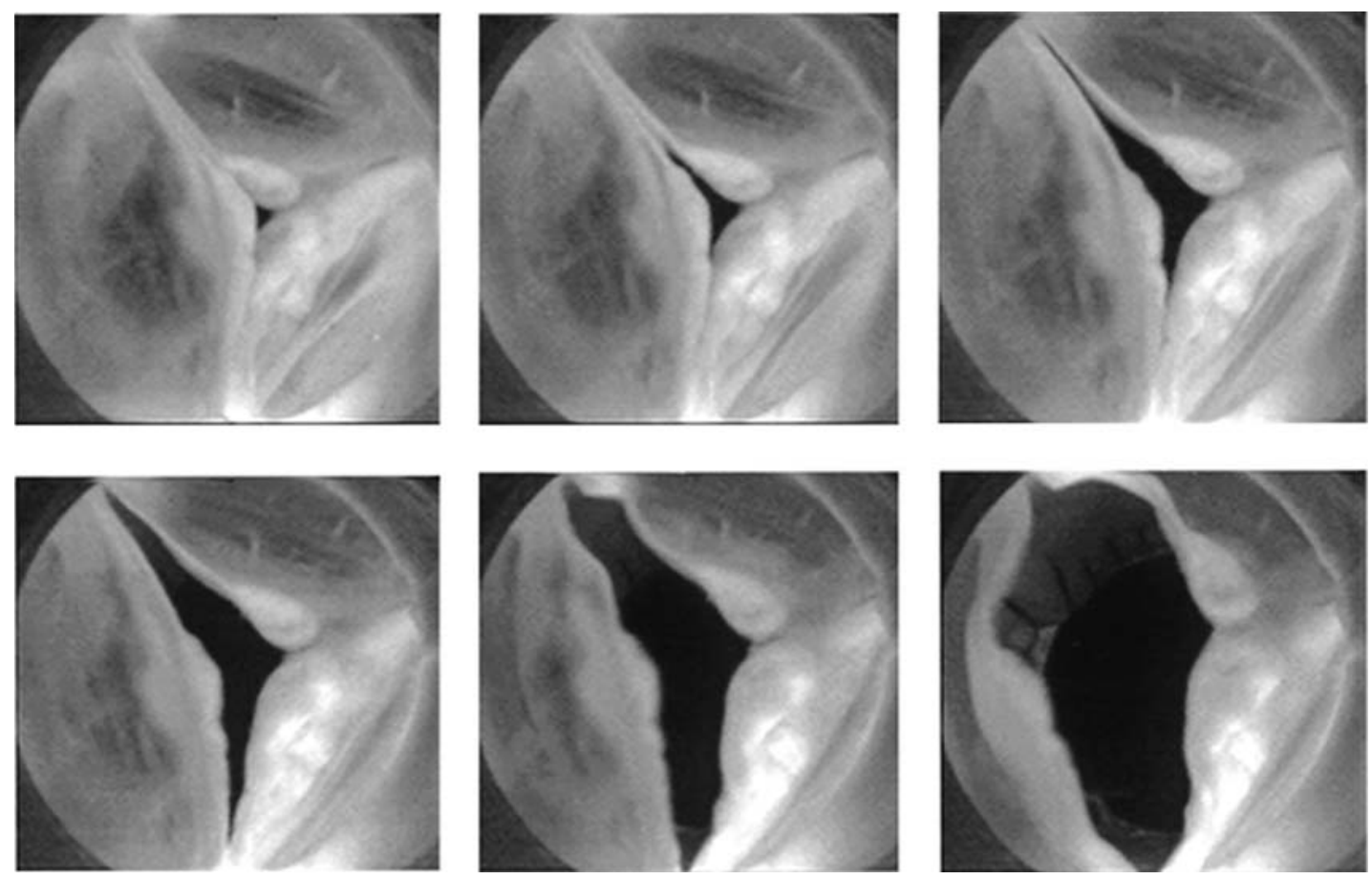

Fig. 3 Opening cycle of a congenitally bicuspid valve obtained from a 18 years old patient, who died of noncardiac causes and had neither symptoms nor measurable aortic valve gradient. Note: At maximum opening, the aortic valve orifice is still 30" stenotic. (Reproduced with permission from Francis Robicsek, Mano J Thubrikar, Joseph W Cook, Brett Fowler. The congenitally bicuspid aortic valve: how does it function? Why does it fail? Ann Thorac Surg 2004;77(1):177-185.)
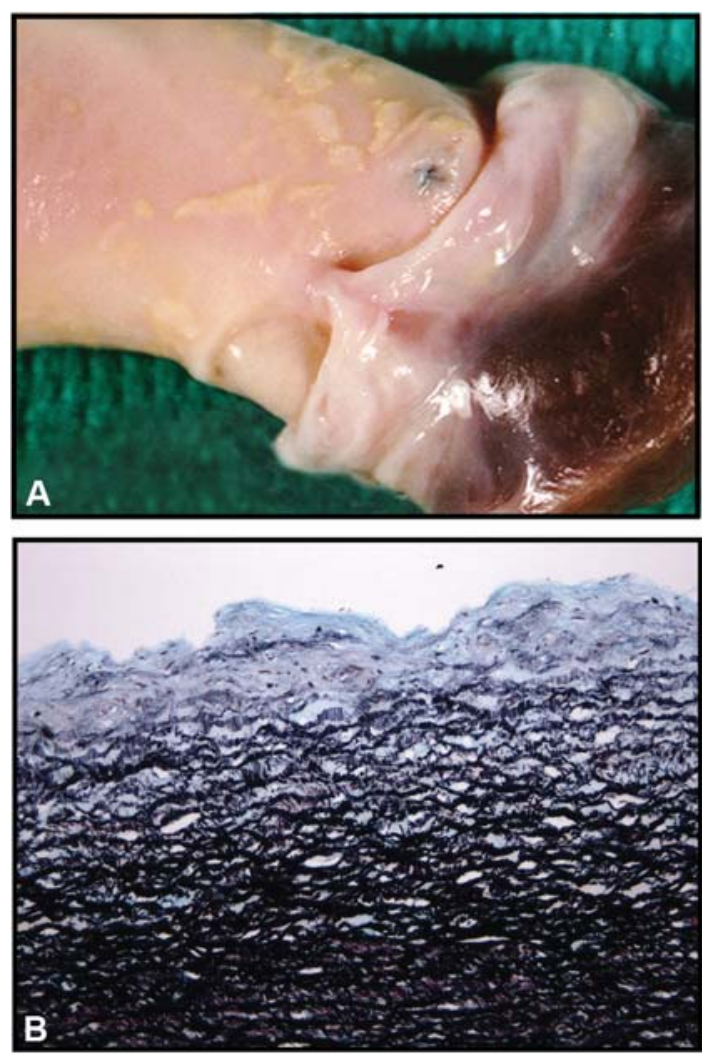

Fig. 4 Visual image (A) and histologic picture (B) (elastic staining) of the ascending aorta of a 5 years old boy with "normal" bicuspid aortic valve who died of noncardiac causes. Note the plaque on the aortic wall caused by "jet-damage" and the disintegration of the elastic fibers on histology. (Adapted from Robicsek et al. ${ }^{14}$ ) (B) $25 x$ magnification. Movat pentachrome stain. this group, the histological examination showed a completely normal ascending aortic wall.

Group 2 consisted of two infants who-besides the bicuspid aortic valve-had other cardiovascular anomalies as well. One infant had hypoplastic left ventricle, and the other had hypoplastic left ventricle and coarctation. The histological examination of the ascending aorta in both infants confirmed disruption of the elastic fibers.

Group 3. The two controls with normal aortic valves revealed no abnormalities in the wall of the ascending aorta (-Fig. 5).

\section{Conclusions}

Because of the extreme difficulties in obtaining newborn cadaver hearts with bicuspid aortic valves, our observations are limited to a very few samples. This is a shortcoming of the study. Our findings, however, reinforce our previous postulates which are as follows:

Patients with CBAVs may lack symptoms and transvalvular pressure gradients, but all bicuspid aortic valves are inherently, anatomically stenotic to a various degree.

Patients with such "normally" functioning aortic valves may develop pathological changes and poststenotic dilatation of the ascending aorta. In patients, where CBAV is the sole anomaly, the aortic wall is normal at birth.

Apparently, there are several subgroups of bicuspid aortic valves. In patients with additional cardiovascular anomalies, because of inborn weakness of the aortic wall, the dilatation may occur earlier, may be more extensive, and may require more radical replacement of the ascending aorta. 

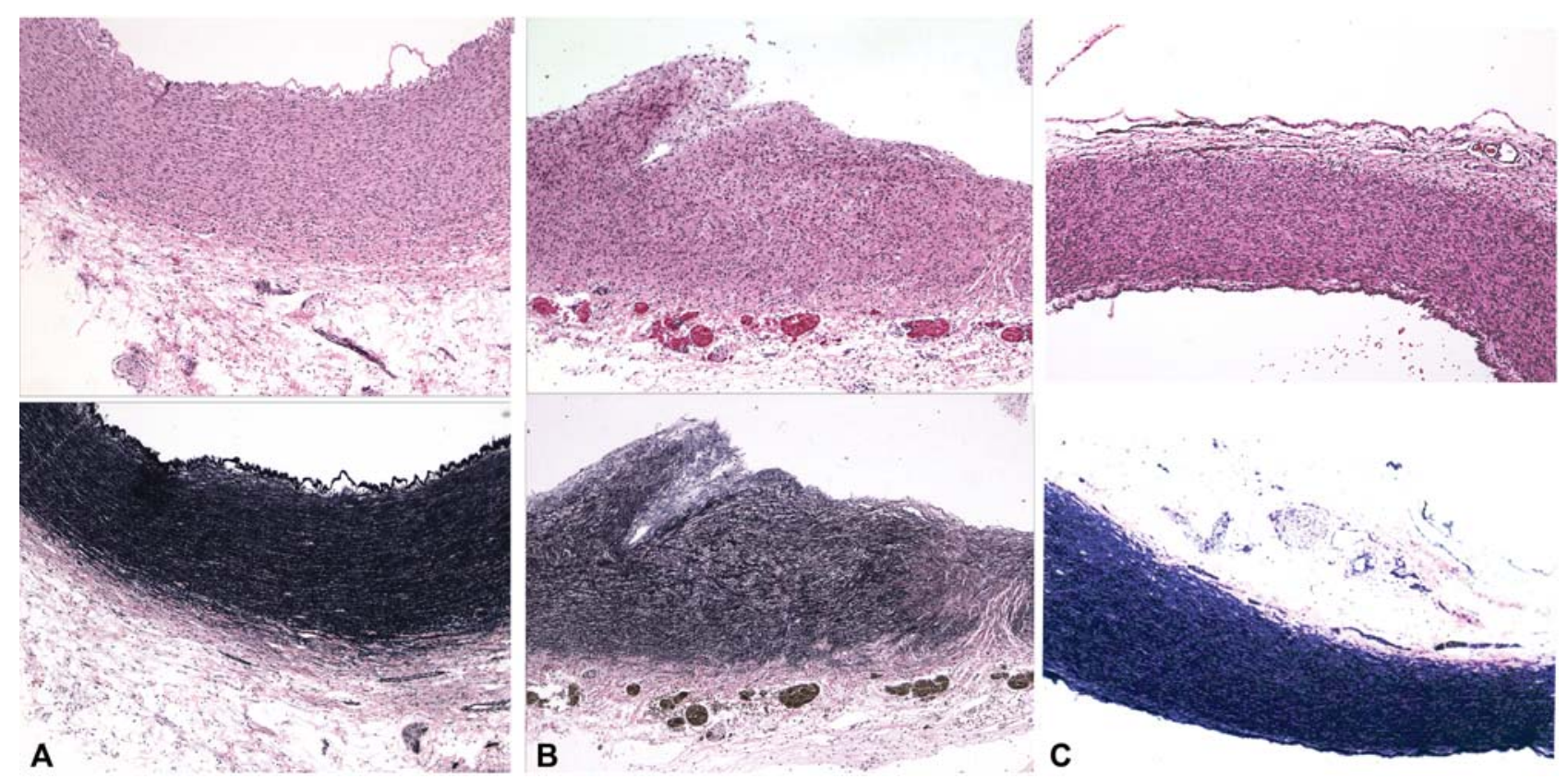

Fig. 5 The histologic pictures of the ascending aortic wall (10X magnification, hematoxylin-eosin and Moffat pentachrome stain) of three newborns with CBAV, who died in the first two weeks of life: Picture $(A)$ is that of a newborn who had CBAV as the sole cardiovascular anomaly. Note that the aortic wall appears to be normal. Picture (B) is that of a newborn who, in addition of CBAV had additional cardiovascular anomalies (hypoplastic left ventricle and coarctation of the aorta). Note that the aortic wall shows disintegration of the normal structure and fragmentation of the elastic fibers. Picture (C) is that of a newborn (control) with normal tri-leaflet aortic valve. The aortic wall, as pictured in A, is entirely normal. (Adapted from Robicsek et $\mathrm{al}^{14}$ ).

In most patients where the bicuspid valve is the only cardiovascular anomaly, dilatation of the ascending aorta may be treated with a less aggressive surgical approach. ${ }^{14,15}$

\section{Conflict of Interest}

None declared.

\section{References}

1 Conti CA, Della Corte A, Votta E, et al. Biomechanical implications of the congenital bicuspid aortic valve: a finite element study of aortic root function from in vivo data. J Thorac Cardiovasc Surg 2010;140(04):890-896, 896.e1-896.e2

2 Verma S, Yanagawa B, Kalra S, et al. Knowledge, attitudes, and practice patterns in surgical management of bicuspid aortopathy: a survey of 100 cardiac surgeons. J Thorac Cardiovasc Surg 2013; 146(05):1033-1040.e4

3 Elefteriades JA. Editorial comment: should aortas in patients with bicuspid aortic valve really be resected at an earlier stage than those in patients with tricuspid valve? Cardiol Clin 2010;28(02):315-316

4 Morgagni JB. The Seats and Causes of Diseases Investigated by Anatomy. Boston, MA: Wells and Lilly; 1824

5 Halsted WS. An experimental study of circumscribed dilatation of an artery immediately distal to a partially occluding band, and its bearing on the dilatation of the subclavian artery observed in certain cases of cervical rib. J Exp Med 1916;24(03):271-286

6 Holman E. The obscure physiology of poststenotic dilatation; its relation to the development of aneurysms. J Thorac Surg 1954;28 (02):109-133
7 Robicsek F. The Post-Stenotic Dilatation of Great Vessels. Acta Morphol;1954

8 Robicsek F. Post-stenotic dilatation of the great vessels. Acta Med Scand 1955;151(06):481-485

9 Robicsek F, Sanger PW, Taylor FH, Magistro R, Foti E. Pathogenesis and significance of post-stenotic dilatation in great vessels. Ann Surg 1958;147(06):835-844

10 Robicsek F, Stamou S. The bicuspid aortic valve. How does it function. Why does it fail? Acta Chir Belg 2007;(3, Supp 107):91

11 Nataatmadja M, West M, West J, et al. Abnormal extracellular matrix protein transport associated with increased apoptosis of vascular smooth muscle cells in Marfan syndrome and bicuspid aortic valve thoracic aortic aneurysm. Circulation 2003;108 (Suppl 1):II329-II334

12 Fedak PW, Verma S, David TE, Leask RL, Weisel RD, Butany J. Clinical and pathophysiological implications of a bicuspid aortic valve. Circulation 2002;106(08):900-904

13 Erbel R, Aboyans V, Boileau C, et al; ESC Committee for Practice Guidelines; The Task Force for the Diagnosis and Treatment of Aortic Diseases of the European Society of Cardiology (ESC). 2014 ESC Guidelines on the diagnosis and treatment of aortic diseases: document covering acute and chronic aortic diseases of the thoracic and abdominal aorta of the adult. Eur Heart J 2014;35 (41):2873-2926

14 Robicsek F, Padera RF, Thubrikar MJ. Dilatation of the ascending aorta in patients with congenitally bicuspid aortic valves. HRS Proc Intensive Care and Cardiovasc Anesth 2012;4(02): $109-118$

15 Robicsek F, Cook JW, Reames MK Sr, Skipper ER. Size reduction ascending aortoplasty: is it dead or alive? J Thorac Cardiovasc Surg 2004;128(04):562-570 